\title{
MaLTESE: Large-Scale Simulation-Driven Machine Learning for Transient Driving Cycles
}

\author{
Shashi M. Aithal and Prasanna Balaprakash \\ Argonne National Laboratory, Lemont, IL, USA \\ \{aithal, pbalapra\}@anl.gov
}

\begin{abstract}
Optimal engine operation during a transient driving cycle is the key to achieving greater fuel economy, engine efficiency, and reduced emissions. In order to achieve continuously optimal engine operation, engine calibration methods use a combination of static correlations obtained from dynamometer tests for steady-state operating points and road and/or track performance data. As the parameter space of control variables, design variable constraints, and objective functions increases, the cost and duration for optimal calibration become prohibitively large. In order to reduce the number of dynamometer tests required for calibrating modern engines, a large-scale simulation-driven machine learning approach is presented in this work. A parallel, fast, robust, physics-based reducedorder engine simulator is used to obtain performance and emission characteristics of engines over a wide range of control parameters under various transient driving conditions (drive cycles). We scale the simulation up to 3,906 nodes of the Theta supercomputer at the Argonne Leadership Computing Facility to generate data required to train a machine learning model. The trained model is then used to predict various engine parameters of interest, and the results are compared with those predicted by the engine simulator. Our results show that a deep-neural-networkbased surrogate model achieves high accuracy: Pearson product-moment correlation values larger than 0.99 and mean absolute percentage error within $1.07 \%$ for various engine parameters such as exhaust temperature, exhaust pressure, nitric oxide, and engine torque. Once trained, the deep-neural-network-based surrogate model is fast for inference: it requires about 16 microseconds for predicting the engine performance and emissions for a single design configuration compared with about 0.5 second per configuration with the engine simulator. Moreover, we demonstrate that transfer learning and retraining can be leveraged to incrementally retrain the surrogate model to cope with new configurations that fall outside the training data space.
\end{abstract}

Keywords: Transient driving cycle modeling - Surrogate modeling - Machine learning $\cdot$ Deep learning $\cdot$ Deep neural networks

\section{Introduction}

In order to achieve the goals of increased fuel economy and performance while reducing emission, automotive manufacturers have implemented various strategies and parameter variables to control and optimize automotive engines. Engine calibration-the process of determining the optimal values of control variables such as spark/fuel injection 
timing, valve timing, exhaust gas recirculation (EGR) fraction) is of paramount importance in achieving high engine performance and fuel economy while meeting emission standards. Currently, to make the problem tractable, automotive manufacturers optimize one or more engine performance indices (e.g., fuel economy, emissions, or engine torque) with respect to a given set of engine-controllable variables such as valve timing, EGR fraction, or ignition/injection timing, with all other conditions such as engine speed and load remaining the same. Optimal values of various engine operating points (speed and load) are obtained via dynamometer tests that are then used to generate engine maps. This procedure is called static calibration for steady-state conditions. These static calibration values are then interpolated to obtain optimal operating conditions for other operating points. The static calibration process, however, presents significant and unique challenges on account of the large design space and conflicting constraints. Over thirty independent design variables, including engine speed (i.e., RPM), torque, air-to-fuel ratio (AFR), and driving conditions (e.g., city or highway) influence the fuel economy, engine performance, and emissions. Moreover, most engines are operated in transient mode, especially during city drives. During the transient mode of operation, the engine speed and load change continuously and frequently (as opposed to a highway drive), and hence optimal operating conditions derived from static calibrations are not accurate. The lack of accuracy stems from the fact that there is a strong nonlinear correlation between various input parameters and outputs. For instance, a small change in the spark timing can increase the engine torque but also greatly increase the NO emission. In order to increase the accuracy for transient engine operation, more calibration tests have to be conducted over a wider range of input/controllable parameters to span the entire feasible engine operating domain. Hence, the cost and duration of the calibration process grow exponentially with the number of input/controllable parameters, greatly increasing the product design cycle/time to market. Even for engines with simple technologies, achievement of the optimal calibrations for the transient driving mode is impractical.

Harnessing the power of high-performance computing, one can perform optimal calibrations for the transient driving conditions using massively parallel computations. Conducting design, analyses, and optimization studies over such a large parameter space presents serious computational challenges, however. To span the entire engine operating range over the vast parameter space requires thousands of combinations of input conditions. For instance, if one were to consider just six different input control parameters with five parametric values for each input variable, one would have 15,625 $\left(5^{6}\right)$ different input combinations for a single transient drive cycle (or commute of a single driver). Given the wide variability in the driving habits of individual drivers and different types of commutes, simulating the typical drives of a handful of drivers would yield over a 100,000 transient simulations. Each such simulation would produce vast amounts of output data, such as peak, average, and cumulative values of emissions, power, engine temperature, and exhaust gas temperature and pressure. Computational time for a typical city or highway drive is also a major barrier to the use of highperformance computing in large-scale transient drive cycle simulations. For instance, the computational time for a single engine cycle (one compression stroke followed by one expansion stroke of the piston) can range from a few hours to days at the strong- 
scaling limit (50-100 cores) of modern multidimensional simulation codes. A typical 25-30-minute drive involves about 40-50,000 engine cycles. Thus, a single multidimensional drive cycle simulation would require well over a year, which precludes their use for such drive cycle simulations and optimization (calibration).

Given the need to simulate typical drive cycles of thousands of vehicles in real time (physical time taken to run engine dynamometer tests or dyno tests) while efficiently harvesting and learning useful design, development, and optimization data, we have developed a modeling framework called MaLTESE ${ }^{1}$ (Machine Learning Tool for Engine Simulations and Experiments). It is a scalable simulation-driven machine learning (ML) framework that enables automotive design engineers to exploit the task parallelism inherent in simulating thousands of transient drive cycles and learning at real-time speeds. The framework also allows the coupling of experimental engine data in order to tune simulation constants and/or train the neural network and hence closely couples largescale simulations, available engine data, and ML. This paper describes the use of MaLTESE to conduct the largest transient driving cycle simulation conducted on the Theta supercomputer at the Argonne Leadership Computing Facility. We also present an indepth study of the use of ML methods to predict engine performance and emissions based on the training and test data obtained from the drive cycle simulations.

The MaLTESE framework consists of two main components: an engine simulator pMODES and a neural-network-based surrogate-modeling tool. Engine simulations of thousands of different typical transient city driving commutes, each approximately 25-30 minutes, were accomplished by using pMODES (parallel Multi-fuel Otto Diesel Engine Simulator). This is a parallel, robust, physics-based real-time engine simulator that can concurrently compute the performance and emissions for thousands of transient drive cycles. The simulator can perform engine simulations for either gasoline (Otto) or diesel engines with any combination of over thirty user-defined input/control variables. Given a set of driving conditions (wind speed, friction, gear-shift/transmission strategy, etc), one can obtain detailed information about over twenty engine outputs, such as fuel consumption, engine performance (power/torque), emissions (carbon dioxide, carbon monoxide, nitric oxide, soot), exhaust gas temperatures and pressures, and maximum engine temperature and pressure. The engine simulator produces the same data as an engine being tested on a dynamometer. Since thousands of driving commutes can be simulated simultaneously, accurate input/output correlations (transient calibration) over a wide range of input parameters can be accomplished without the prohibitive testing costs. Furthermore, since the drive cycle simulations can be conducted at speeds faster than real time, a typical drive cycle simulation can be conducted in less than 30 minutes, hence making it practical for the design and development of fleets of cars. A subset of the large calibration data is then input to the neural-network-based surrogate modeling tool. Based on the calibration data, a surrogate model is trained to capture the relationship between the multiple inputs and outputs. The trained surrogate model can then be used to predict expected calibration values of other driving conditions and can be a part of the engine control unit. Large computing clusters with thousands of cores greatly reduce the wall time and effort by concurrently simulating thousands of driving cycles. A subset of the large data set was generated from over 300 million engine op-

\footnotetext{
${ }^{1}$ aptly named after a small, intelligent dog that loves to learn new tricks
} 
erating points in a typical commute of 250,000 different drivers. Finding the optimum operating condition for a given engine operation (speed, load, driving condition) can improve engine efficiency, reduce emissions, reduce engine wear and tear, and improve fuel economy. Use of large-scale computing and data analytics for drive cycle analyses enables engine designers to reduce the cost and time required for engine dyno tests, hence reducing the product design cycle and cost to consumers.

The main goal of this paper is to use MaLTESE to demonstrate the following:

1. Concurrent simulation of thousands of driving cycles with the engine simulator (pMODES) for a typical 25-minute commute at faster-than-real-time speed

2. Ability of deep neural networks to use a small subset of the parameter space to train a model and predict engine output characteristics of any arbitrary driving cycle in the parameter space

3. Inference time of a deep-neural-network-based surrogate model being considerably lower than simulations with near $1 \%$ error in prediction accuracy.

This paper is organized as follows. Section 2 describes the method of solution for the engine simulation and the training and testing of the neural-network-based ML predictions. Section 3 presents the numerical experiments using various ML methods. Section 4 discusses related work. The main conclusions of the paper are presented in Section 5.

\section{Surrogate modeling for transient drive cycle simulation}

In this section, we discuss the engine simulator and the ML approach for surrogate modeling. We also describe the parameters of the drive cycle simulations and the choice of the parameter subspace to train the neural network.

\subsection{Engine simulator}

The engine simulator pMODES [2,3] is used to compute the temporal variation of various engine parameters such as pressure, temperature, and mixture composition for each CAD over an entire drive cycle. The energy equation shown in Eq. (1) describes the relationship between the engine crank angle $\theta$ and instantaneous pressure $(P(\theta)$ ).

$$
\frac{d P(\theta)}{d \theta}=\frac{\gamma-1}{V(\theta)}\left(Q_{\text {in }}-Q_{\text {loss }}\right)-\gamma \frac{P(\theta)}{V(\theta)} \frac{d V}{d \theta}
$$

Here, $Q_{i n}$ is the heat input due to fuel combustion, $Q_{\text {loss }}$ is the heat lost from the engine, $\gamma$ is the ratio of specific heats of the working fluid, and $V(\theta)$ is the instantaneous volume of the cylinder. Solution of this equation yields the temporal variation of cylinder pressure for a given set of operating conditions (such as load, combustion duration, fuel type, and engine RPM). The instantaneous values of temperature and composition of the burned and unburned gas zones can be obtained from the instantaneous value of computed pressure. Knowing the instantaneous temperature, pressure, and composition of the burned zone, one can compute emissions such as nitric oxide, carbon monoxide, soot, and unburned hydrocarbons using simplified reduced chemistry models. Details of 
these models and the solution procedure are discussed in Ref. [4]. Instantaneous values of equilibrium concentrations of the combustion products are needed in order to compute various emissions. Computation of these equilibrium concentrations poses serious numerical challenges because of the stiffness of the system of nonlinear equations describing the formation of combustion products. References [2,3] discuss the details of the computation procedure and steps taken to ensure a fast, robust solution. Following the solution procedure discussed above, one can obtain a temporal variation of output quantities such as emissions ( $\mathrm{NO}, \mathrm{CO})$, engine exhaust temperature and pressure, and torque as a function of time. Figure 1 in Ref. [5] shows the temporal variation of NO and $\mathrm{CO}$ for a given fuel injection pattern.

In this work we considered sixteen driving cycles. Each transient cycle had 1,500 data points corresponding to a typical 25 -minute commute, with data sampled every second $(25 * 60)$. For each drive cycle, we considered five values for six independent engine parameters - spark timing, engine rpm (depends on gear ratio), ambient air temperature, air humidity, internal EGR fraction (proportional to valve timing), and compression ratio (engine size) - thus yielding 15,625 cases $\left(5^{6}\right)$ with different input conditions for each drive cycle and 250,000 for all sixteen drive cycles considered. This number of 250,000 drive cycles is representative of the rush-hour traffic on four major freeways in a typical large city.

\subsection{ML-based surrogate modeling}

A class of ML approaches used for surrogate modeling is supervised learning [6]. Typically, it is used to model the relationship between the output variables and several independent input variables. In this work, we seek to find a surrogate model that captures the relationship between the five output variables (exhaust temperature, exhaust pressure, $\mathrm{NO}, \mathrm{CO}$, and engine torque) and the ten input variables (ambient air temperature, air humidity, valve timing, engine size, spark timing, gear ratio, fuel injection rate, air-fuel ratio, engine inlet pressure, and intake air mass). A supervised learning method takes as input a set $\mathcal{T}$ of $N$ training points of the form $\left\{\left(x_{1}, y_{1}\right), \ldots,\left(x_{N}, y_{N}\right)\right\}$, where $x_{i}$ and $y_{i}$ are the input and output vectors of the $i$ th training point, respectively. The training procedure of the supervised learning method seeks to find a surrogate function $h$ for $f: X \rightarrow Y$, where $f$ is an unknown function that maps the multidimensional input space $X$ to the multidimensional output space $Y$, respectively, such that the difference between $f\left(x_{i}\right)$ and $h\left(x_{i}\right)$ is minimal for all $x_{i} \in \mathcal{T} \subset \mathcal{D}$, where $\mathcal{D}$ is the full data set.

Arguably, classical ML methods are limited in their ability to learn directly from raw data. For decades, the development of ML surrogate models required considerable domain expertise to transform raw input data into a suitable internal representation from which the system could try to learn the relationship between inputs and outputs. Recently, representation learning methods have been developed to automatically discover representations that are best for learning the relationship between inputs and outputs [14]. Deep learning approaches [18] are representation learning methods with multiple levels of representation. They are obtained by composing simple nonlinear computational units that transform the representation at one level into a representation at a higher, slightly more abstract level. These approaches have dramatically improved 


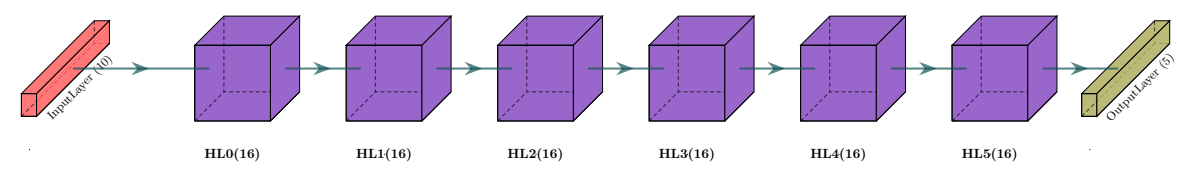

Fig. 1: The dnn configuration obtained for transient drive cycle surrogate modeling

the state of the art in many ML tasks, such as speech recognition, visual object recognition, drug discovery, and genomics [14,18].

Deep neural network $(\mathrm{dnn})[18]$ systems are a prominent class of deep learning approaches. A dnn comprises a stack of computational layers organized in a hierarchical way, with the layers connected through a system of weighted connections. Each layer has a number of simple computational units, each with a nonlinear transformation operation called an activation function. The input layer of the $d n n$ receives a batch of input data, which is transformed into higher-level representations through the stack of computational layers and weighted connections. The output layer of the dnn gives the predicted values of the outputs. During the training phase, the weights of the connections in the network are adjusted to minimize prediction errors. This adjustment is achieved efficiently by using a back propagation method that calculates the gradient of the error with respect to all the weights in the network and uses it in a stochastic gradient-based optimization to adjust the connection weights.

While there exists a standard dnn configuration for traditional ML tasks such as image and text classification, there is no default or general-purpose dnn configuration for surrogate modeling of engineering applications and in particular transient drive cycle modeling. Designing a suitable dnn for a given modeling task is a key research challenge for many nontraditional ML tasks.

We carried out an exploratory study and developed a relatively simple multilayered feed-forward neural network. Figure 1 shows the obtained network used in this work: the input layer of size $|X|=10$ is connected to a dense hidden layer with 16 units (HLO) and a rectified linear activation function (not shown in the figure). This configuration is repeated six times (HL1,.., HL5), where the output of the previous layer is given as the input for the next layer. Consequently, the 16 units of the $j$ th layer are connected to each of the 16 units in the $(j+1)$ th layer. The last layer is the output layer of size $|Y|=5$ that gives predicted values.

\section{Experimental results}

In this section, we first describe the setup that we used to assess the efficacy of the proposed dnn method. We then describe the training data generation and prediction accuracy results.

\subsection{Setup}

In addition to dnn, many classical ML methods (sometimes referred to as shallow learning methods) for surrogate modeling exist in the literature. Based on the algorith- 
mic similarity and functionality, they can be grouped as regularization, instance-based, recursive partitioning, kernel-based, bagging, and boosting methods. For comparison with dnn, we selected several classical ML methods to cover different groups: ridge regression ( $\mathrm{rg}$ ) [16], $k$-nearest-neighbor regression $(\mathrm{knn})$ [6], support vector machine $(\mathrm{svm})$ [25], decision tree $(\mathrm{dt})$ [19], random forest $(r f r)$ [8], extremely randomized trees (etr) [13], ADA-boosting regression $(a b r)$ [11], bagging regression $(b r)$ [7 [ 20], gradient boosting regression (gbr) [12], and eXtreme gradient boosting (xgb) [9]. As a baseline, we also included the simplest regression method, multivariate linear regression $(\mathrm{lm})$.

The ML training and inference experiments were run on a single-node hardware platform with a $3.4 \mathrm{GHz}$ Intel Xeon E5-2687W processor (8 cores per CPU), $64 \mathrm{~GB}$ RAM, with an NVIDIA Tesla P100, 16 GB GPU RAM. The dnn training and inference leveraged GPUs, whereas the classical ML methods used only the host CPU processors.

We used Python (Intel distribution, version 3.6.3) and the scikit-learn library [23] (version 0.19.0) to implement all the classical ML methods. We used the default hyperparameters provided by the scikit-learn library for the ML methods. For dnn, we used Keras [10] (version 2.0.8), a high-level neural network Python library that runs on the top of the TensorFlow library [1] (version 1.3.0). We used the following hyperparameter settings for $d n n$ training: epochs $=50$, batch size $=16$, loss $=$ mean squared error, and optimizer=adam. While dnn natively supports multioutput regression, where we can build a single model with multiple outputs, the classical ML methods considered in our study do not support multioutput regression. Therefore, we built one model for each output. We leveraged the MultioutputRegressor interface in the scikit-learn library to build the multioutput regression models.

Given the different ranges for inputs and outputs, ML methods benefit from preprocessing the training and the testing data set. For each input and output, we applied MinMaxScaler and StandardScaler transformations in the scikit-learn library. The former scales the values between 0 and 1 , and the latter removes the mean and scales the values to unit variance. We applied the two transformations before training and applied the inverse of StandardScaler and MinMaxScaler transformations after inference so that evaluation metrics were computed on the original scale. Note that the inverse transformations are required only for the predicted output values.

We adopted two evaluation metrics to assess the accuracy of the ML models on the test data and to compare them. The first metric is the Pearson product-moment correlation coefficient $(r)$, which we use to measure the strength of a linear association between observed and predicted values on the test data. This metric ranges from -1 to +1 . A value of 1 indicates a perfect linear relationship between observed and predicted values. A value of 0 indicates that no linear correlation exists between observed and predicted values and thus the prediction accuracy of the model is poor. A value of less than 0 means that as the value of observed (predicted) values increases, the value of the predicted (observed) values decreases. While this metric does not capture the absolute error, it is particularly useful when engineers build ML models for optimization as an end goal, where the relative ordering of the predicted values is sufficient to choose the best configurations. The second metric is the mean absolute percentage error (MAPE) given by the mean of $100 \times \frac{\left|y^{i}-\hat{y}^{i}\right|}{y_{i}} \%$ for $i \in 1, \ldots, n$, where $y_{i}$ and $\hat{y}_{i}$ are observed 
and predicted values of the test data point $i$, respectively. We used this metric to assess the prediction error for each output.

\subsection{Training data generation at scale}

As explained earlier, 250,000 different transient drive cycles were simulated concurrently by using the engine simulator pMODES to generate the training and test data for the ML algorithms. The simulations were conducted on Theta-a 4,392-node, 11.69petaflop Cray XC40-based leadership-class supercomputer at the Argonne Leadership Computing Facility (ALCF). Each node of Theta is a 64-core Intel Xeon Phi processor with 16 gigabytes of high-bandwidth in-package memory, 192 GB of DDR4 memory, and a 128 GB SSD. The nodes of Theta are interconnected by an Aries fabric. Theta has a total file system capacity of 10 petabytes.

In this work, large-scale computing was used to exploit the inherent task parallelism in the simulation of a large number of drive cycles. In such applications, it is important to demonstrate that the overall size of the problem (number of drive cycles considered) does not adversely affect the total wall time for simulation. In order to test the weakscaling characteristics of the simulation, three different tests were run, with 62,500 , 12,5000 , and 250,000 cases run concurrently, corresponding to $1 / 4,1 / 2$, and near-fullmachine simulation (3,906 nodes out of 4,392 nodes). Since each drive cycle was run concurrently on a single processor, the total wall time for each of these cases should be nearly constant. Within each set of runs, the simulation time for an individual drive cycles depends on the computations required for the emissions, which can vary depending on the case being considered. Table 1 shows the minimum and maximum time required for computing an individual drive cycle.

Table 1: Weak Scaling on Theta

\begin{tabular}{|c|c|c|}
\hline \hline Nodes (cases) & Min Time (sec) & Max Time (sec) \\
\hline $1024(62500)$ & 728 & 1157 \\
\hline $2048(125000)$ & 740 & 1252 \\
\hline $3906(250000)$ & 720 & 947 \\
\hline \hline
\end{tabular}

The runtime for a typical 25-minute drive cycle was about 12-15 minutes (faster than real time) on the Intel Knights Landing (KNL) cores on Theta. From Table 1, we can see that the minimum simulation time is nearly constant for all the cases considered. We also can see that the maximum simulation time is nearly constant for the $1 / 4$ and $1 / 2$ machine size cases, whereas the near-full-machine simulation is about $20 \%$ lower. The system load from other jobs on the machine seems to have a greater impact on the simulation time for cases $1 \& 2$ compared with case 3 , where there is less interference from other jobs on the system. These results demonstrate that one can simulate drive cycles of various sizes - even thousands-on a large-scale production cluster such as Theta without a serious penalty on overall wall time for computation as the size of the drive cycle simulations increases. Such a capability might be required if one were to use 
physics-based models to develop the acceleration and braking strategies for connected vehicles in order to optimize fuel efficiency, reduce emissions, and reduce engine wear and tear.

The discussion also shows that conducting a drive cycle simulation of a large parameter space requires considerable computational resources. To minimize the use of large-scale computing for drive cycle analyses, we investigated the possibility of using machine learning techniques wherein a small subset of the large parameter space is used as training data. We explored the possibility of using a trained model in predicting the characteristics of other drive cycles without the need for conducting simulations or gathering engine data with acceptable accuracy. In this work, we generated the following data sets for training and testing.

1. train-data-1: From the complete parametric study of 250,000 different drive cycles, a representative set of 64 different drive cycles, spanning the input parameter range, was chosen for training data. We used Latin hypercube sampling [21], a statistical method on the input space to select the 64 representative sets. Since each drive cycle had 1,500 data points for fuel flow rate, a total of 96,000 data points were used for training.

2. test-data-1a: To test the accuracy of prediction from the training set, we used two different test data sets from the entire set of 250,000 (excluding those used in the training). The first set comprised four different drive cycles, which had the same fuel flow rate but for which the other input parameters were different, for a total of 6,000 data points.

3. test-data-1b: In addition to this test data set, a random set of four drive cycles (for a total of 6,000 points) was chosen for the 250,000 cases (excluding those used to train the model). By random, unlike the test-data-1a case, no parameter was intentionally kept constant.

4. test-data-2: Both the test cases test-data-1a and test-data-1b were drive cycles wherein the range of input parameters of the test drive cycles was the same as that for the training model. In order to test the efficacy of the ML methods wherein the test data might have parameters beyond the bounds of the trained data, a third data set was generated. This data set had a fuel flow rate that was $20 \%$ higher than the corresponding fuel flow rate used in test-data-1a. Furthermore, the engine RPM was lower than that used in test-data-1a by $17 \%$.

\subsection{Comparison of ML methods}

In this section, we compare the different ML methods that were trained on train-data-1 and tested on test-dat $a-1 \mathrm{a}$ and test-dat $\mathrm{a}-1 \mathrm{~b}$. We use parallel coordinate plots to visualize the accuracy metrics obtained by the ML methods on the five outputs. In the plot, each output is given an axis; the five axes are placed parallel to each other. Each axis can have a different scale because each output can have a different range of values. Given an ML method, its accuracy value on each axis is connected and visualized through a line.

The parallel coordinate plots for Pearson product-moment correlation coefficients are shown in Figures $2 \mathrm{a}$ and $2 \mathrm{~b}$. On both testing data sets, all ML methods obtain correlation coefficients larger than 0.92 . On test-data-1a, dnn outperforms other ML 


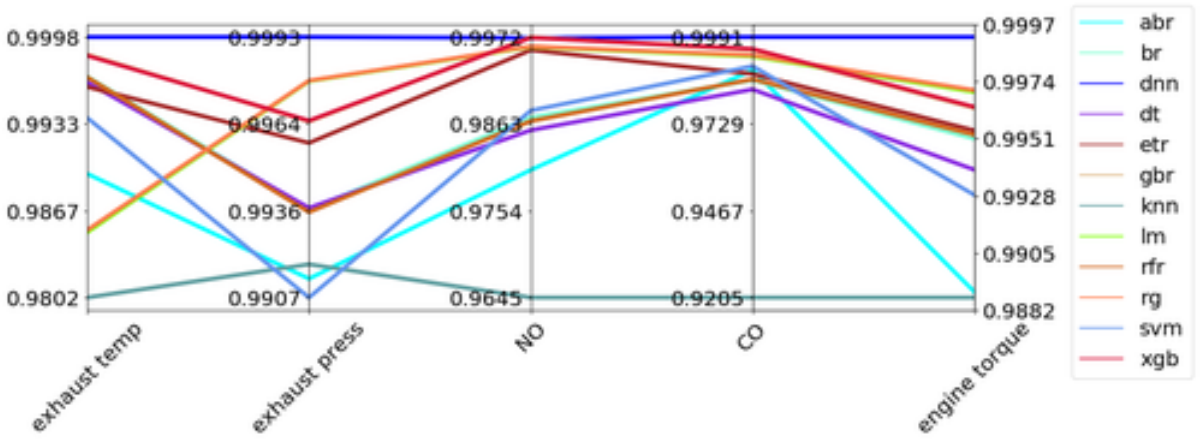

(a) Pearson product-moment correlation coefficients on test-data-1a

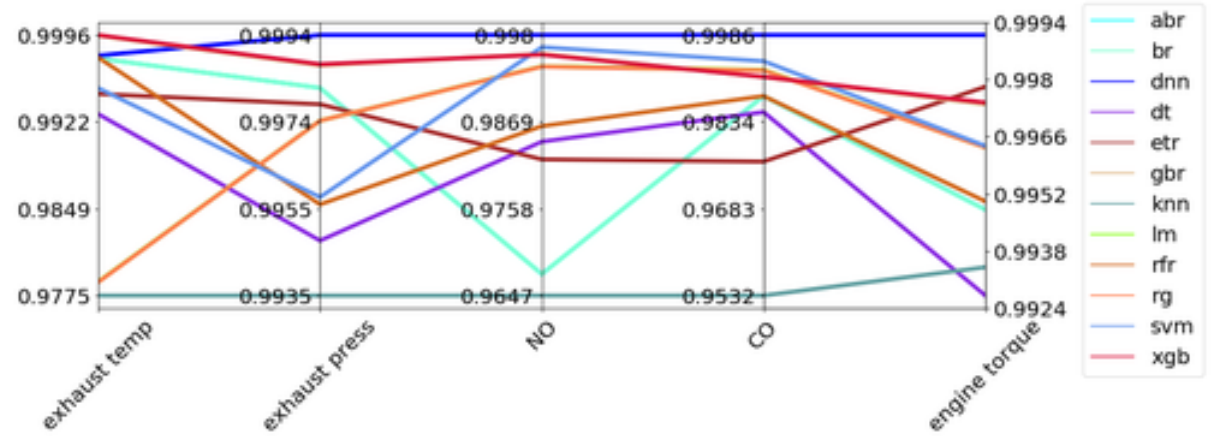

(b) Pearson product-moment correlation coefficients on test-data-1b

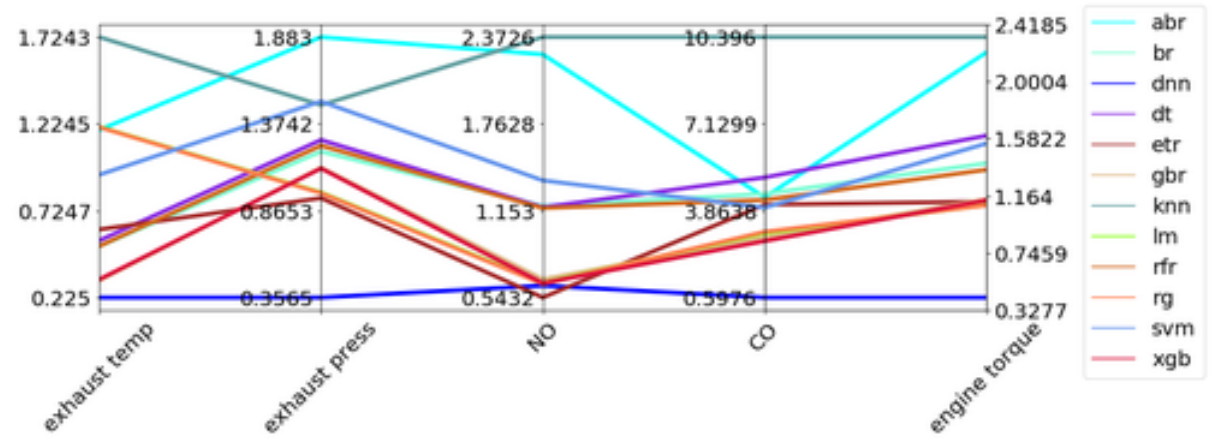

(c) Mean absolute percentage errors (MAPE) on test-data-1a

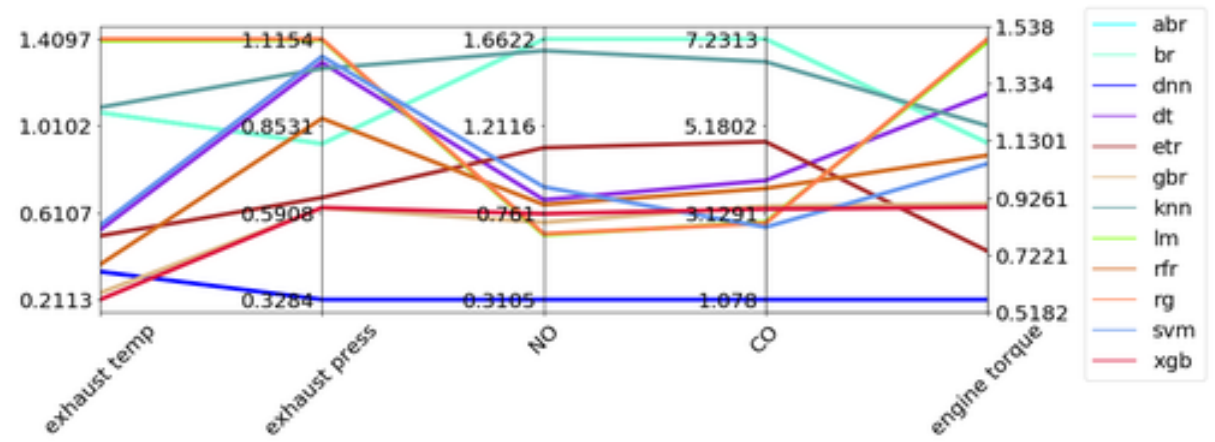

(d) Mean absolute percentage errors (MAPE) on test-data-1b

Fig. 2: Parallel coordinate plot showing the accuracy metrics obtained by different ML methods 
methods, obtaining correlation coefficient values larger than 0.99 for exhaust temperature, exhaust pressure, $\mathrm{NO}, \mathrm{CO}$, and engine torque, respectively. The trend is similar on test-data-1b, where dnn achieves larger correlation coefficient values than those of the classical ML methods. An exception is for exhaust temperature, where the correlation coefficient of $\mathrm{xgb}$ is slightly larger than $\mathrm{dnn}$.

Figures $2 \mathrm{c}$ and $2 \mathrm{~d}$ show the MAPE values on test-data-1a and test-data-1b, respectively. The range of error percentages for exhaust temperature, exhaust pressure, $\mathrm{NO}$, and engine torque is between $0.2 \%$ and $2.5 \%$; but for $\mathrm{CO}$ the error goes up to $10.39 \%$ and $7.23 \%$ on test-data-1a and test-data-1b, respectively. This indicates that prediction of $\mathrm{CO}$ is more difficult than prediction of exhaust temperature, exhaust pressure, NO, and engine torque. The MAPE values obtained by dnn are smaller than those of other ML methods. In particular, dnn achieves significantly smaller MAPE values for the outputs; exhaust pressure, $\mathrm{CO}$, and engine torque. Overall, MAPE values of dnn are not more than $0.59 \%$ and $1.07 \%$ on test-data-1a and test-data-1b, respectively. The scatter plots of observed and predicted values from dnn for the five outputs on test-data-1a and test-data-1b are given in Appendix A.
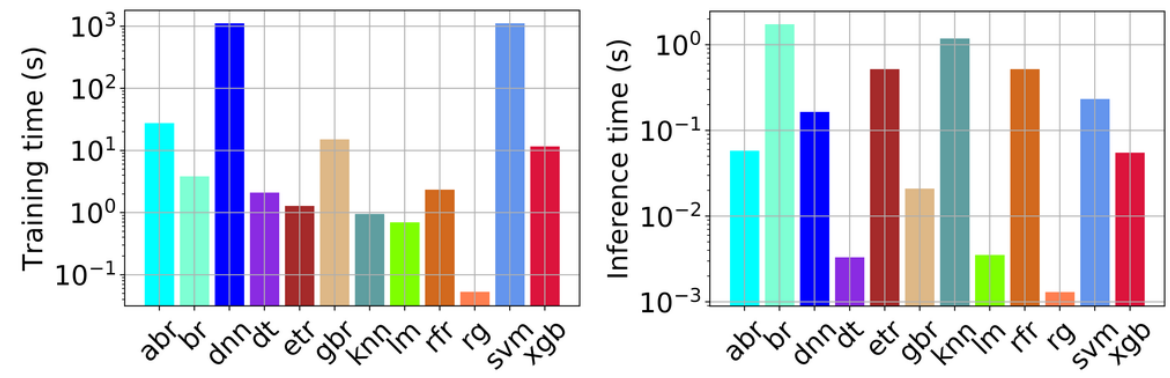

(a) Time required for training different ML (b) Time required for inference using different methods with train-data-1 (96000 train- ML methods on test-data-1a (6000 testing points) ing points)

Fig. 3: Bar plots showing the training and inference times of the different ML methods

Figure 3 shows the training and inference times of the different ML methods on train-data-1 and test-data-1a, respectively. From 3a, we observe that dnn requires approximately 1,000 seconds for training. On the other hand, the training times of classical ML methods range between 0.1 and 10 seconds. An exception is svm, which requires a training time similar to that of dnn. Even though dnn leverages P100 GPUs, it is more computationally expensive than other ML methods. This difference can be attributed to the cubic algorithmic time complexity. The bagging and boosting methods typically have a time complexity of $O(N \log N)$ in the training set size $N$. Figure $3 b$ shows the time required for inference on test-data-1a. To predict 6,000 points, dnn requires approximately 0.1 seconds ( 16 microseconds/configuration), which is lower than that of several sophisticated classical ML methods such as br, 


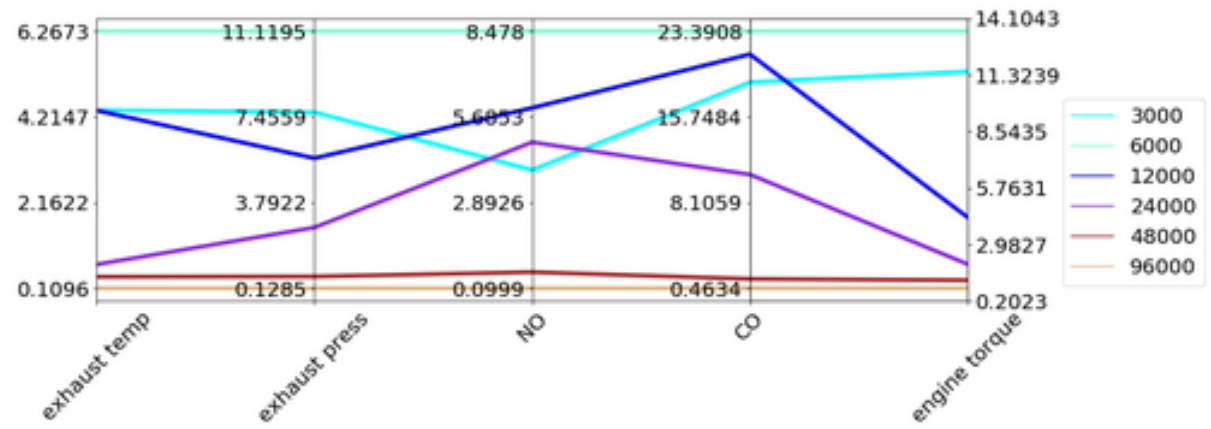

Fig. 4: Parallel coordinate plot showing the impact of training data set size on the MAPE values obtained by dnn on test-data-1a

etr, $r f r$, and svm. Simple ML methods such as $l m, r g$, and dt require less than 0.01 seconds, but their accuracy values are not as high. We observed a similar trend on test-data-1b.

\subsection{Impact of training set size}

We studied the impact of the training data size on the accuracy of the dnn method by varying the number of training points. In addition to the default training data size of 96,000 , we considered training set sizes of 1,500, 3,000,6,000, 12,000, 24,000 and 48,000 data points (1,500 data points represent one complete transient drive cycle; hence, the training set sizes represent $1,2,4,8,16$, and 32 different drive cycles). For each training set size, we trained the dnn method and evaluated the model on test-data-1a.

The results are shown in Figure 4, where the lines in the parallel coordinate plot correspond to the training data set sizes. We observe that an increase in the training set size decreases the MAPE values. We did not include the MAPE values for 1,500 because the error values are too high (greater than 100\%), which results in skewed axes ranges. The dnn model trained with 3,000 points yields MAPE values between 6.26\% and $23.39 \%$. While the MAPE values for training set size to 6,000 and 12,000 are lower than that of 3,000, to achieve MAPE values within $1 \%$ for all the outputs, the dnn model requires at least 48,000 training points.

\subsection{Model adaptation using transfer learning and retraining}

We evaluated the efficacy of the ML models when the test data falls outside the training data regime. We took the ML models trained on train-data-1 and tested them on test-data-2. The results are shown in Figure 5a. The ranges of MAPE values for all ML models are large: [1.71\%, 20.24\%] for exhaust temperature, [10.21\%, 22.73\%] for exhaust pressure, $[1.83 \%, 15.25 \%]$ for $\mathrm{NO},[10.61 \%, 269.43 \%]$ for $\mathrm{CO},[5.34 \%$, 


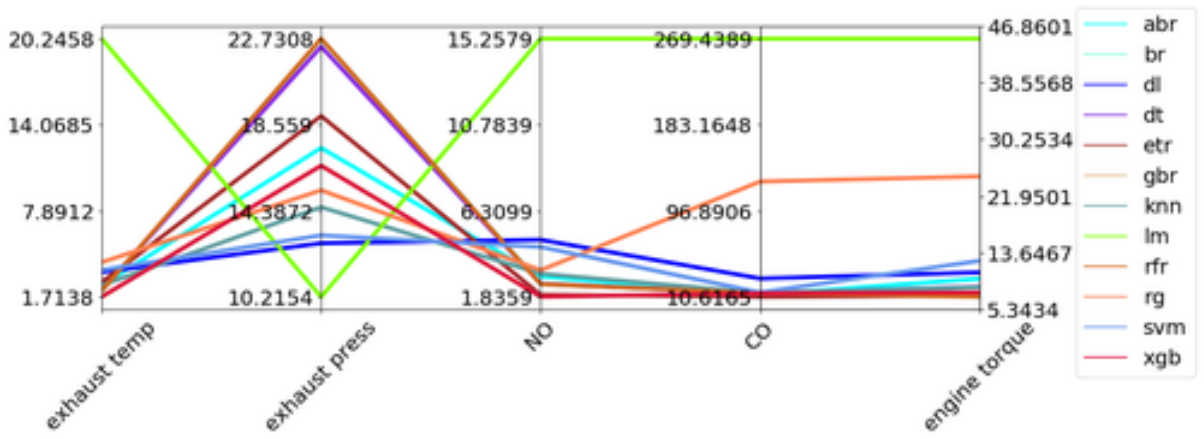

(a) MAPE values obtained by the ML methods on test-data-2

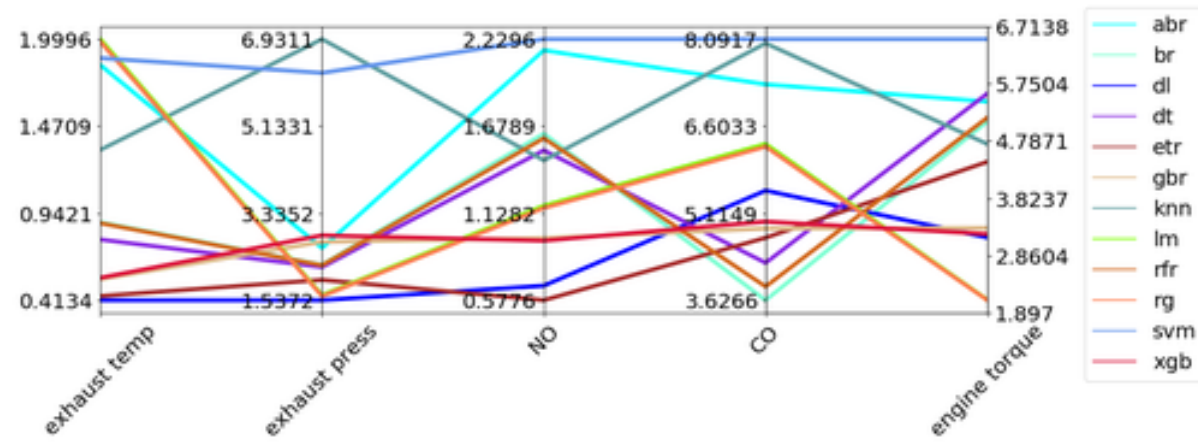

(b) MAPE values obtained by the ML methods on test-data-2 with retraining and transfer learning

Fig. 5: Parallel coordinate plots showing the impact of retraining and transfer learning on the ML methods

46.86\%] for engine torque. This range can be attributed to the fact that while ML methods can generalize the learned functional relationship inside the input space spanned by the training points, outside that space their prediction power decreases significantly.

A promising approach to adapt ML models for new test cases such as test-data-2 involves calibrating the trained model by using transfer learning, where a model trained on one task can be adapted to a similar task with limited training data. In our case, a small subset of data from test-data-2 can be used to retrain the model. Nevertheless, not all ML methods offer that transfer learning capability. Among the ML methods considered in our study only dnn can be used for transfer learning. All other methods require complete retraining, where one needs to add the new data to the training data and train from scratch.

We used 1,500 points from test-data-2 for transfer learning and retraining from scratch. We note that that the training data set size of 1,500 points alone resulted in poor prediction accuracy on train-data-1. Our hypothesis is that using the ideas 
of transfer learning and retraining but with the same limited data setting, we can significantly improve the prediction accuracy of the ML models.

For $d n n$, we took the model trained on train-data-1. To enable transfer learning, we froze the weights of HiddenLayer0, HiddenLayer1, and HiddenLayer2 layers (see Figure 1); used 1,500 points from test-data-2; and retrained the dnn model, where the weights of HiddenLayer3, HiddenLayer4, and HiddenLayer5 layers were adjusted. The retrained dnn model was then used to predict the outputs in test-data-2. For other ML models, we used the retraining-from-scratch approach, where we added 1,500 points from test-data-2 to 96,000 points of train-data-1 and trained the ML models.

The results are shown in Figure 5b. We observe that both the transfer learning method and training from scratch for other ML methods significantly reduce error values for all the outputs. The ranges of MAPE values are $[0.41 \%, 1.99 \%]$ for exhaust temperature, $[1.53 \%, 6.93 \%]$ for exhaust pressure, $[0.57 \%, 2.22 \%]$ for NO, $[3.62 \%$, $8.09 \%$ ] for $\mathrm{CO}$, and $[1.89 \%, 6.71 \%]$ for engine torque. The dnn method obtains smaller MAPE values for three outputs: $0.41 \%$ for exhaust temperature, $1.53 \%$ for exhaust pressure, and $0.58 \%$ for NO. Only for CO and engine torque are the MAPE values larger. Overall, however, MAPE is within 5.5\%.

\section{Related work}

Given the importance of internal combustion (IC) engines in transportation and power generation, considerable work has been conducted in the area of predicting their performance and emissions, including the use of soft computing techniques such as artificial neutral networks (ANNs). Some of the earliest attempts to use ANNs to predict the NO emissions during the transient operation of a diesel engine were reported by Ref. [17]. Similar attempts were reported in Refs. [15] and [22]. Reference [24] provides an exhaustive review of the use of soft computing techniques in automotive engines. All the reported results with these techniques for IC engines are for a single engine/transient operation. Most of these reported results use experimental data from an engine operation with a small set of input parameters (two to three input variables) to predict a specific output quantity such as NO or exhaust temperature. None of the papers have attempted to use machine learning techniques to predict the performance and emissions of a fleet of cars with a large set of input parameters, each of which was varied over a large operating range, as reported in this work.

To the best of our knowledge, this is the single largest drive cycle simulation $(250,000$ different cases) conducted by using a well-validated, physics-based reduced-order model at faster-than-real-time computing speeds. This is also the first demonstration of the ability to apply ML methods to such large-scale engine data to predict performance and emissions.

\section{Conclusion}

We developed MaLTESE, a simulation-driven machine learning modeling framework that couples massively parallel simulations of thousands of engine drive cycles at real- 
time speeds and a machine-learning-based surrogate modeling approach. We scaled the simulation up to 3,906 nodes on the Theta supercomputer at the Argonne Leadership Computing Facility to generate data for training the surrogate model. We developed a deep-neural-network-based surrogate model and compared it with several classical machine learning methods. From our numerical experiments we observed that all learning methods yielded reasonably good prediction accuracy. We demonstrated that the deep neural network is a promising method: it outperforms other classical machine learning techniques and achieves correlation coefficient values larger than 0.99 and a mean absolute percentage error within $1.07 \%$ for exhaust temperature, exhaust pressure, nitric oxide, carbon monoxide, and engine torque. Our comparative study of machine learning methods provides valuable input to design engineers who can make an informed decision about the use of machine learning methods for their design and development assessments.

In addition to the prediction accuracy of various machine learning methods, we studied the training and inference times for the various learning methods. We observed that the training time for deep neural networks was about two to four orders of magnitude higher than that for classical machine learning methods: $0.1-10 \mathrm{~s}$ for classical methods vs $1000 \mathrm{~s}$ for deep neural networks. Once the model is trained, however, the interference time required by the deep neural network to predict the output characteristics 6,000 data points (4 different drive cycles) is about 0.1 seconds ( 16 microseconds/configuration). As shown earlier, concurrent simulation of four different drive cycles on four KNL processors would take over 700 seconds. These inference timing studies show that the deep-neural-network-based surrogate-model can be used for real-time control using the emerging low-cost and relatively low-powered on-board deep learning chips.

The parametric study of the size of the training set showed that for predicting all output variables within 1\% accuracy, 48,000 data points (corresponding to 32 different representative drive cycles) were required. This study shows that a small subset of wellchosen representative drive-cycles (64 drive cycles in this case) can be used to predict the output of other drive cycles without having to simulate the entire parametric range (250,000 drive cycles). Based on the transfer learning studies, we have demonstrated the possibility of using machine learning methods to yield high-accuracy prediction even when the input parameter space is considerably different from the parameter range used for training.

\section{Acknowledgment}

This research used resources of the Argonne Leadership Computing Facility, which is a DOE Office of Science User Facility supported under Contract DE-AC02-06CH11357. This material was based upon work supported by the U.S. Department of Energy, Office of Science, under Contract DE-AC02-06CH11357.

\section{References}

1. Abadi, M., Barham, P., Chen, J., Chen, Z., Davis, A., Dean, J., Devin, M., Ghemawat, S., Irving, G., Isard, M., et al.: Tensorflow: a system for large-scale machine learning. In: OSDI. vol. 16, pp. 265-283 (2016) 
2. Aithal, S.M.: Analysis of the current signature in a constant-volume combustion chamber. Combustion Science and Technology 185(2), 336-349 (2013). https://doi.org/10.1080/00102202.2012.718297

3. Aithal, S.M.: Prediction of voltage signature in a homogeneous charge compression ignition (HCCI) engine fueled with propane and acetylene. Combustion Science and Technology 185(8), 1184-1201 (2013). https://doi.org/10.1080/00102202.2013.781593

4. Aithal, S.M.: Development of an integrated design tool for real-time analyses of performance and emissions in engines powered by alternative fuels. In: Proc. SAE 11th Int. Conf. Engines \& Vehicles (2013). https://doi.org/10.4271/2013-24-0134, SAE Paper 2013-24-0134

5. Aithal, S.M., Wild, S.M.: ACCOLADES: A scalable workflow framework for large-scale simulation and analyses of automotive engines. In: International Conference on High Performance Computing. pp. 87-95. Springer (2015)

6. Bishop, C.M.: Pattern Recognition and Machine Learning, vol. 1. Springer, New York (2006)

7. Breiman, L.: Bagging predictors. Machine learning 24(2), 123-140 (1996)

8. Breiman, L.: Random forests. Mach. Learn. 45(1), 5-32 (2001)

9. Chen, T., Guestrin, C.: Xgboost: A scalable tree boosting system. arXiv preprint arXiv:1603.02754 (2016)

10. Chollet, F., et al.: Keras. https://keras.io(2015)

11. Drucker, H.: Improving regressors using boosting techniques. In: ICML. vol. 97, pp. 107115 (1997)

12. Friedman, J.H.: Stochastic gradient boosting. Comput. Stat. Data Anal. 38(4), 367-378 (2002)

13. Geurts, P., Ernst, D., Wehenkel, L.: Extremely randomized trees. Machine learning 63(1), 3-42 (2006)

14. Goodfellow, I., Bengio, Y., Courville, A., Bengio, Y.: Deep learning, vol. 1. MIT press Cambridge (2016)

15. Hashemi, N., Clark, N.: Artificial neural network as a predictive tool for emissions from heavy-duty diesel vehicles in southern california. International Journal of Engine Research 8(4), 321-336 (2007)

16. Hoerl, A.E., Kennard, R.W.: Ridge regression: Biased estimation for nonorthogonal problems. Technometrics 12(1), 55-67 (1970)

17. Krijnsen, H.C., van Kooten, W.E., Calis, H.P.A., Verbeek, R.P., Bleek, C.M.v.d.: Prediction of NOx emissions from a transiently operating diesel engine using an artificial neural network. Chemical Engineering \& Technology: Industrial Chemistry-Plant Equipment-Process Engineering-Biotechnology 22(7), 601-607 (1999)

18. LeCun, Y., Bengio, Y., Hinton, G.: Deep learning. Nature 521(7553), 436-444 (2015)

19. Loh, W.Y.: Classification and regression trees. Wiley Interdisciplinary Reviews: Data Mining and Knowledge Discovery 1(1), 14-23 (2011)

20. Louppe, G., Geurts, P.: Ensembles on random patches. In: Joint European Conference on Machine Learning and Knowledge Discovery in Databases. pp. 346-361. Springer (2012)

21. McKay, M., Beckman, R., Conover, W.: Comparison the three methods for selecting values of input variable in the analysis of output from a computer code. Technometrics; (United States) 21 (5 1979). https://doi.org/10.1080/00401706.1979.10489755

22. Parlak, A., Islamoglu, Y., Yasar, H., Egrisogut, A.: Application of artificial neural network to predict specific fuel consumption and exhaust temperature for a diesel engine. Applied Thermal Engineering 26(8-9), 824-828 (2006)

23. Pedregosa, F., Varoquaux, G., Gramfort, A., Michel, V., Thirion, B., Grisel, O., Blondel, M., Prettenhofer, P., Weiss, R., Dubourg, V., Vanderplas, J., Passos, A., Cournapeau, D., Brucher, M., Perrot, M., Duchesnay, E.: Scikit-learn: Machine learning in Python. Journal of Machine Learning Research 12, 2825-2830 (2011) 
24. Shrivastava, N., Khan, Z.M.: Application of soft computing in the field of internal combustion engines: A review. Archives of Computational Methods in Engineering 25(3), 707-726 (2018)

25. Smola, A.J., Schölkopf, B.: A tutorial on support vector regression. Statistics and Computing 14(3), 199-222 (2004)

\section{Appendix A}

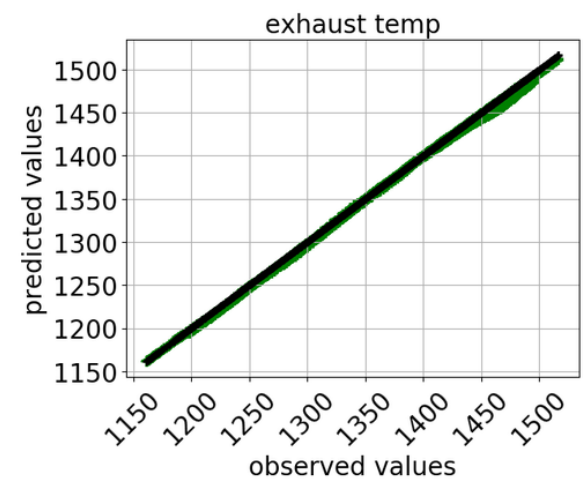

(a) test-data-1a

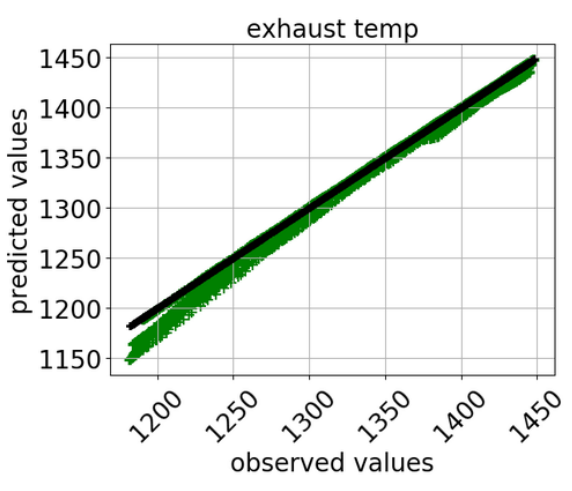

(b) test-data-1b

Fig. 6: Scatter plot of observed and predicted values from dnn for exhaust temperature on test-data-1a and test-data-1b

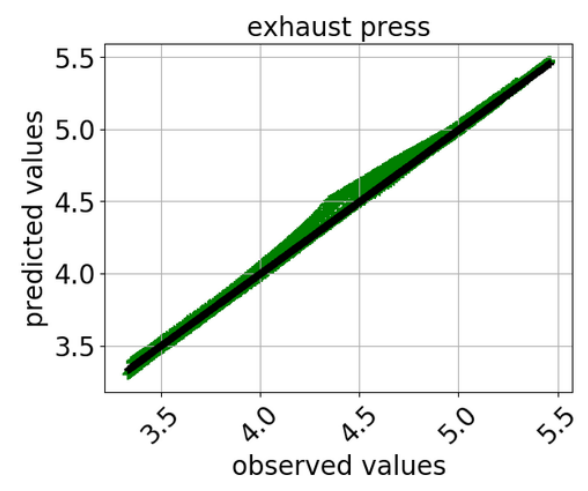

(a) test-data-1a

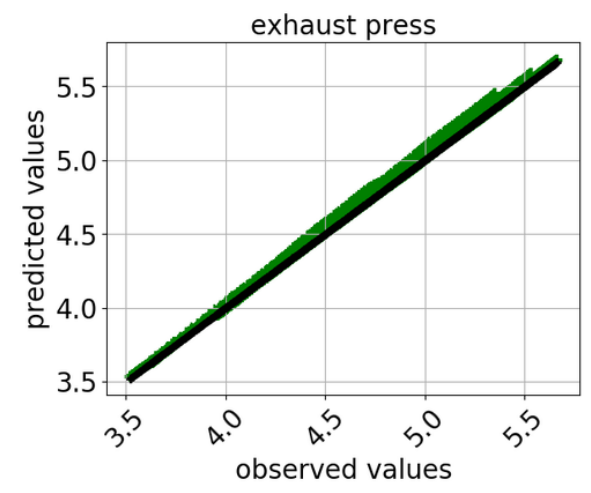

(b) test-data-1b

Fig. 7: Scatter plot of observed and predicted values from dnn for exhaust pressure on test-data-1a and test-data-1b 


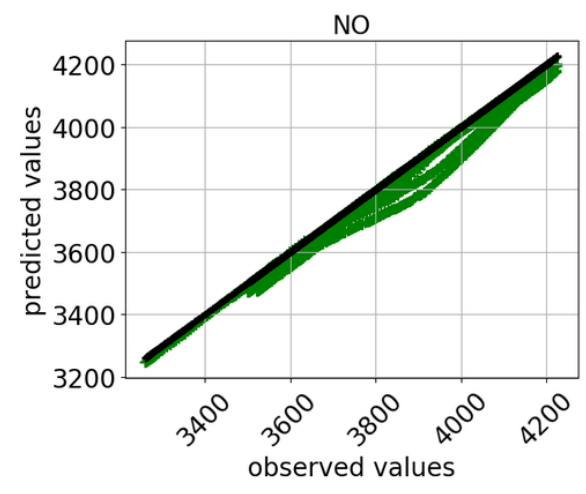

(a) test-data-1a

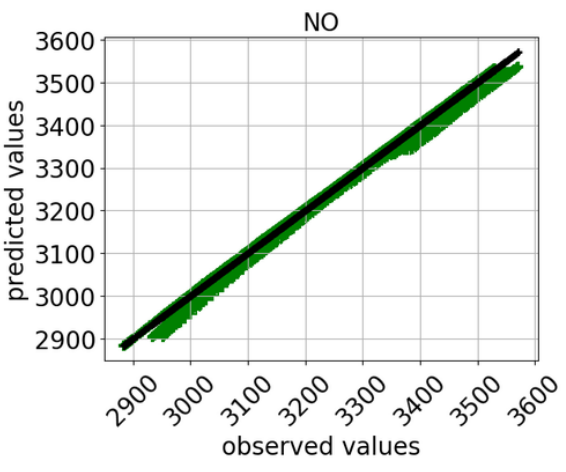

(b) test-data-1b

Fig. 8: Scatter plot of observed and predicted values from dnn for NO on test-data-1a and test-data-1b

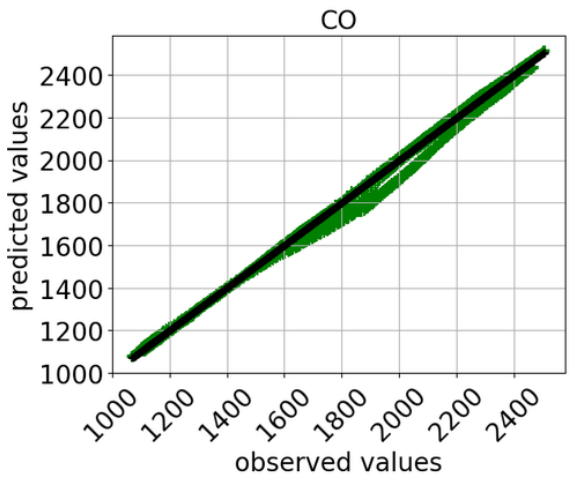

(a) test-data-1a

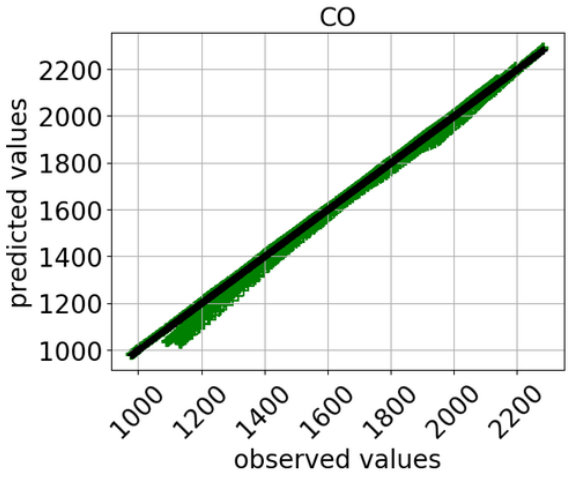

(b) test-data-1b

Fig. 9: Scatter plot of observed and predicted values from dnn for $\mathrm{CO}$ on test-data-1a and test-data-1b

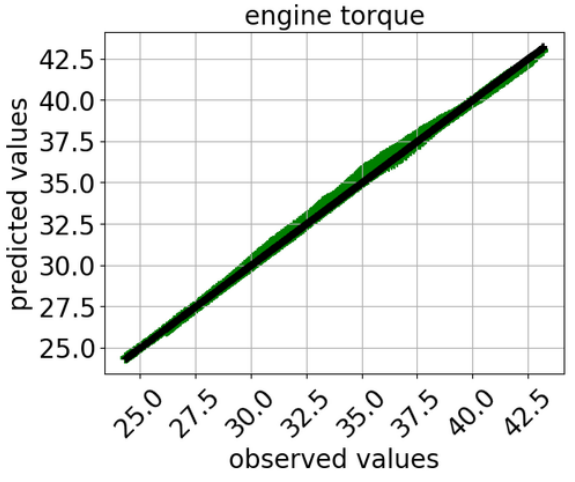

(a) test-data-1a

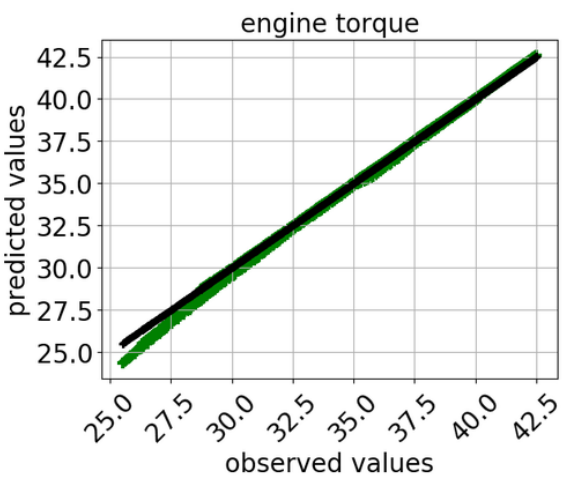

(b) test-data-1b

Fig. 10: Scatter plot of observed and predicted values from dnn for engine torque on test-data-1a and test-data-1b 